01

\title{
Динамика фотоиндуцированного вращения сферической частицы в постоянном электрическом поле
}

\author{
(C) А.И. Грачев \\ Физико-технический институт им. А.Ф.Иофффе РАН, \\ 194021 Санкт-Петербург, Россия \\ e-mail: grach.shuv@mail.ioffe.ru
}

(Поступило в Редакцию 13 января 2018 г.)

\begin{abstract}
Проанализирована динамика описанного ранее явления вращения в постоянном электрическом поле сферической частицы в условиях ее освещения, индуцирующего электрический дипольный момент сферы. Представлено общее рассмотрение динамики эффекта, позволившее уточнить условия реализации режима адиабатического вращения. Особенности неадиабатического вращения частицы продемонстрированы на примере сферы, помещенной в среду с бесконечно малой вязкостью. Показано, что динамика неадиабатического вращения в существенной степени определяется соотношением между величиной электрои фотоиндуцированных дипольных моментов сферы. Также представлены результаты краткого анализа динамики вращения частицы в случае небольшого отклонения ее формы от сферической.
\end{abstract}

DOI: 10.21883/JTF.2019.01.46953.16-18

\section{Введение}

В недавно опубликованной работе [1] впервые было описано явление вращения в постоянном однородном электрическом поле сферической частицы, в которой в условиях ее постоянного освещения непрерывно генерируется электрический дипольный момент, ортогональный направлению силовых линий поля. Были проанализированы конкретные фотоэлектрические механизмы генерации дипольного момента, удовлетворяющие симметрии данного явления. Фотоиндуцированное вращение в отличие от близкого к нему явления, вращения Квинке [2], свободно от основного недостатка последнего - спонтанности направления оси вращения частицы. Это позволяет надеяться, что рассматриваемый эффект позволит продвинуться по пути создания микрои наномоторов, а также различных устройств, например, насосов для целей микро- и нанофлюидики.

Целью настоящей работы является дальнейший анализ эффекта фотоиндуцированного вращения, конкретно, его динамики. Полученные результаты позволили уточнить критерии введенного в [1] понятия режима адиабатического вращения сферической частицы, реализующегося при наличии достаточно сильного механического сопротивления со стороны окружающей сферу среды. При незначительности указанного сопротивления вращение становится неадиабатическим, а его динамика, в общем случае, носит сложный характер, требующий учета зависимости фото- и электрически индуцированных дипольных моментов от угловой скорости вращения. Представлены результаты анализа влияния на динамику вращение частицы нарушения экспериментальных условий, принятых в [1], прежде всего отклонения ее формы от сферической.

\section{Общее рассмотрение}

Общая геометрия эксперимента по фотоиндуцированному вращению описана в [1] (рис. 1). Сфера фотоактивного материала радиуса $R$ помещена в диэлектрическую жидкость, находящуюся в однородном постоянном электрическом поле $\mathbf{E}_{\mathbf{0}}$. На сферу падает пучок света, направление распространения которого отвечает вектору $\mathbf{k} \perp \mathbf{E}_{0}$. Свет генерирует в направлении $\mathbf{k}$ электрический дипольный момент $\mathbf{p}_{\mathrm{ph}}$, взаимодействие которого с электрическим полем индуцирует момент вращения $\mathbf{T}_{\mathrm{ph}}$ :

$$
\mathbf{T}_{\mathrm{ph}}=\mathbf{p}_{\mathrm{ph}} \times \mathbf{E}_{0} .
$$

В [1] рассматривался только режим стационарного вращения сферы, определяемый условием равенства $\mathbf{T}_{\mathrm{ph}}$ моменту $\mathbf{T}_{\text {liq }}$, воздействующему на вращающуюся сферу со стороны вязкой диэлектрической жидкости, который в первом приближении пропорционален угловой скорости вращения $\omega$. Предполагалось, что скорость стационарного вращения $\omega_{m}$ удовлетворяет условию, так называемого адиабатического вращения: $\omega_{m} \tau_{\text {rel }} \ll 1$ (где $\tau_{\text {rel }}-$ характерное время релаксации $\mathbf{p}_{\mathrm{ph}}$ ), когда величина и направление фотогенерируемого дипольного момента вращающейся сферы совпадает с $\mathbf{p}_{\mathrm{ph}}$. При условии выполнения указанного неравенства и по отношению к дипольному моменту $\mathbf{p}_{\mathrm{el}}$, индуцируемому $\mathbf{E}_{0}$, предполагалось, что в случае $p_{\text {ph }} \ll p_{\text {el }}$ выполняется также неравенство $\omega_{m} \tau_{\text {rel }} \ll p_{\text {ph }} / p_{\text {el }}$.

В общем случае динамика движения сферы описывается следующим уравнением:

$$
d \mathbf{L}(t) / d t=J(d \omega(t) / d t)=\mathbf{T}_{\mathrm{ph}}(\omega)-\mathbf{T}_{\mathrm{liq}}(\omega)-\mathbf{T}_{\mathrm{el}}(\omega),
$$

где $\mathbf{L}$ и $\mathbf{J}$ - момент количества движения и момент инерции сферы соответственно, $\omega$ - вектор угловой скорости. Зависимость $\mathbf{T}_{\mathrm{ph}}$ от $\omega$, как и наличие члена 
$\mathbf{T}_{\mathrm{el}}(\boldsymbol{\omega})$, обусловленного дипольным моментом $\mathbf{p}_{\mathrm{el}}$, отвечает случаю реализации неадиабатического вращения частицы [1]. Для определения вида $\mathbf{T}_{\mathrm{el}}(\boldsymbol{\omega})$ можно воспользоваться результатами феноменологического описания вращение Квинке, базирующегося на представлении об эффективном дипольном моменте сферы $\tilde{\mathbf{p}}_{\mathrm{el}}(\omega)[3,4]$. Аналогичным образом можно поступить и в отношении $\mathbf{p}_{\text {ph }}$, вводя эффективный дипольный момент $\tilde{\mathbf{p}}_{\mathrm{el}}(\omega)$. Однако это требует решения задачи об установлении дрейфо-диффузионного равновесия внутри вращающейся сферы для конкретного механизма фотогенерации $\mathbf{p}_{\mathrm{ph}}$. Насколько нам известно на данный момент постановка подобной задачи в физике твердого тела (физике полупроводников) отсутствует, хотя имеется пример решения близкой задачи - моделирования фотоэлектрического поведения фотовольтаической ячейки сферической формы [5]. Поэтому на данный момент можно лишь уточнить условия перехода от адиабатического вращения к неадиабатическому в той части, что касается выполнения соотношения $\omega_{m} \tau_{\text {rel }} \ll p_{\text {ph }} / p_{\text {el }}$. Как нетрудно понять, указанное неравенство может быть удовлетворено лишь при условии, что $\mathbf{T}_{\mathrm{el}}(\omega)<<\mathbf{T}_{\text {liq }}(\omega)$ при любых значениях $\omega$ вплоть до $\omega_{m}$. Для определения момента $\mathbf{T}_{\mathrm{el}}(\omega)$, действующего в наших экспериментальных условиях, воспользуемся выражением (11) в [3], в результате получим

$$
\mathbf{T}_{\mathrm{el}}(\omega)=12 \pi R^{3} \varepsilon_{0} \varepsilon_{l} E_{0}^{2}\left(\varepsilon_{l} / \varepsilon_{s}+2 \varepsilon_{l}\right)\left[\omega \tau_{r} / 1+\left(\omega \tau_{r}\right)^{2}\right],
$$

где $\varepsilon_{0}$ - диэлектрическая постоянная вакуума, $\varepsilon_{l}$ и $\varepsilon_{s}$ - относительная диэлектрическая проницаемость жидкости и материала сферы соответственно, а $\tau_{r}=\varepsilon_{0}\left(\varepsilon_{s}+2 \varepsilon_{l}\right) / \sigma_{s}$, где $\sigma_{s}-$ фотопроводимость сферы. Теперь, используя выражения для $\mathbf{T}_{\text {liq }}$ и $\mathbf{p}_{\mathrm{ph}}$ ((2) и (7) в [1]) и предполагая стационарное вращение сферы со скоростью $\omega_{m}=\varepsilon_{0} \varepsilon_{s} E_{\mathrm{ph}} E_{0} / 2 \eta$, неравенство $\mathbf{T}_{\text {el }}(\omega) \ll \mathbf{T}_{\text {liq }}(\omega)$ можно представить следующим обра3ом:

$$
3\left(E_{0} / E_{\mathrm{ph}}\right)\left[\varepsilon_{l}^{2} / \varepsilon_{s}\left(\varepsilon_{s}+2 \varepsilon_{l}\right)\right]\left[\omega_{m} \tau_{r} / 1+\left(\omega_{m} \tau_{r}\right)^{2}\right] \ll 1,
$$

где $E_{\mathrm{ph}}$ - величина фотоиндуцированного электрического поля сферы, а $\eta$ - динамическая вязкость жидкости. Поскольку без потери общности можно полагать выполненным соотношение $\tau_{r} \sim \tau_{\text {rel }}$, то при реализации условия $\omega_{m} \tau_{\text {rel }} \ll 1$ неравенство (4) приобретет следующий вид:

$$
3 E_{0}^{2}\left(\varepsilon_{0} \varepsilon_{l}\right)^{2} / 2 \eta \sigma_{s} \ll 1 .
$$

$\mathrm{C}$ другой стороны, используя известное выражение для $p_{\mathrm{el}}$, приведенное, например, в [6] и предполагая, что $\tau_{\text {rel }} \sim \varepsilon_{0} \varepsilon_{s} / \sigma_{s}$, неравенство $\omega_{m} \tau_{\text {rel }} \ll p_{\text {ph }} / p_{\text {el }}$ можно выразить следующим образом:

$$
E_{0}^{2} \varepsilon_{0}^{2} \varepsilon_{s} \varepsilon_{l} / 2 \eta \sigma_{s} \ll 1 .
$$

Сравнивая (5) и (6), видим, что они совпадают, если $\varepsilon_{s}=3 \varepsilon_{l}$. Однако в реальных экспериментальных условиях следует ожидать, что $\varepsilon_{s}$ будет превосходить величину $\varepsilon_{l}$ более чем на порядок, поэтому неравенство (6) является более сильным.
Предполагая выполненными оба условия адиабатичности, рассмотрим случай вращения освещенной сферы после мгновенной подачи электрического напряжения, тогда уравнение динамики будет иметь теперь следующий вид:

$$
J(d \omega(t) / d t)=\mathbf{T}_{\mathrm{ph}}-\gamma \boldsymbol{\omega},
$$

где $\gamma$ - коэффициент трения вращения сферы в жидкой среде. Используя известные выражения для $J$ и $\gamma$, получаем

$$
d \omega(t) / d t=\omega_{m} \tau_{i}^{-1}-\omega(t) \tau_{i}^{-1},
$$

где $\tau_{i}=J / \gamma=(20 \pi \eta R / m)^{-1}$ - постоянная времени, отражающая степень инерции вращения, и $m-$ масса сферы. Для оценки величины $\tau_{i}$ используем следующие значения параметров: $R \sim 0.1 \mu \mathrm{m}, m \sim 2 \cdot 10^{-17} \mathrm{~kg}$ ( $m=(4 / 3) \pi R^{3} d$, где $d \sim 5 \cdot 10^{3} \mathrm{~kg} \cdot \mathrm{m}^{-3}$ - удельный вес материала сферы), и $\eta \sim 10^{-2} \mathrm{~Pa} \cdot \mathrm{s}$, в результате получим $\tau_{i} \sim 3 \cdot 10^{-10} \mathrm{~s}$.

Полученная оценка означает, что в реальных экспериментальных условиях инерция вращения может проявиться явным образом лишь при использовании электрических или лазерных импульсов с длительностью фронтов не превышающих $\tau_{i}$. В последнем случае характерное время релаксации фотопроводимости частицы и $\mathbf{p}_{\mathrm{ph}}$ (т.е. $\left.\tau_{\mathrm{rel}}\right)$ также должны быть ниже значений $\tau_{i}$.

\section{Динамика неадиабатического вращения}

Как видно из полученных выше результатов, при заданном значение $E_{0}$ уменьшение вязкости диэлектрической жидкости рано или поздно приведет к переходу к режиму неадиабатического вращения. Особенности последнего наиболее наглядно можно проиллюстрировать, рассматривая случай вращения в среде с вязкостью, в пределе стремящейся к нулю, например, сферу, левитирующую в вакууме. Поскольку в этом случае $\mathbf{T}_{\text {el }}(\omega) \ll \mathbf{T}_{\text {liq }}(\omega)$, уравнение динамики сферы приобретет следующий вид:

$$
J(d \omega(t) / d t)=\mathbf{T}_{\mathrm{ph}}(\omega)-\mathbf{T}_{\mathrm{el}}(\omega) .
$$

В отличие от вращения Квинке (являющегося в рамках развиваемых представлений примером неадиабатического вращения) фотоиндуцируемое вращение сферы реализуется при любых значениях электрического поля, поэтому решение (9) следует проанализировать при различных соотношениях между величинами $p_{\mathrm{ph}}$ и $p_{\mathrm{el}}$.

1) $p_{\text {ph }} \ll p_{\text {el. }}$ В этом случае возможна реализация режима вращения, когда условие адиабатичности выполняется для $\mathbf{p}_{\mathrm{ph}}\left(\omega_{m} \tau_{\mathrm{rel}} \ll 1\right)$, но, естественно, нарушено для $\mathbf{p}_{\mathrm{el}}$. Тогда (9) приобретает вид, аналогичный уравнению (7), и с использованием полученных выше результатов его можно представить следующим образом:

$$
J(d \omega(t) / d t)=4 \pi R^{3} \varepsilon_{0} \varepsilon_{s} E_{\mathrm{ph}} E_{0}\left[1-3\left(E_{0} / E_{\mathrm{ph}}\right) \varepsilon_{s}^{-2} \omega \tau_{\mathrm{rel}}\right] .
$$


В условиях стационарного вращения выражение в квадратных скобках в правой стороне (10) равно нулю. Предполагая, например, что $\omega_{m} \tau_{\mathrm{rel}} \leq 10^{-2}$, получим следующее условие реализации данного режима вращения $E_{0} / E_{\mathrm{ph}} \geq 30 \varepsilon_{s}^{2}$.

Из сравнения (10) с уравнением (7) следует, что динамика вращения также подчиняется экспоненциальной зависимости с $\tau_{i}$ обратно пропорциональным $E_{0}^{2}$.

2) $p_{\text {ph }} \sim p_{\text {el. }}$. Этот режим отвечает окончательному нарушению обоих условий адиабатического вращения, поэтому, как уже говорилось выше, уравнение (9) необходимо дополнить уравнением, описывающим кинетику установления дрейфо-диффузионного равновесия внутри вращающейся сферы, позволяющим определить ориентацию $\tilde{\mathbf{p}}_{\text {ph }}$ и конкретный вид зависимости его величины от $\omega$.

3) $p_{\mathrm{ph}} \gg p_{\mathrm{el}}$. Динамика вращения сферы будет определяться конкретным видом зависимости $\mathbf{T}_{\mathrm{ph}}(\omega)$, при этом значение $\omega_{m}$ будет не от величины $\tilde{\mathbf{p}}_{\mathrm{ph}}\left(\omega_{m}\right)$, а главным образом от его ориентации, т.е. угла $\zeta$ между

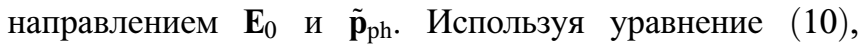
получим следующее выражение для $\sin \xi\left(\omega_{m}\right)$ :

$$
\sin \zeta\left(\omega_{m}\right)=3\left[E_{0} / E_{\mathrm{ph}}\left(\omega_{m}\right)\right]\left(1 / \varepsilon_{s}\right)^{2}\left[\omega_{m} \tau_{\mathrm{rel}} / 1+\left(\omega_{m} \tau_{r}\right)^{2}\right]
$$

где $E_{\mathrm{ph}}\left(\omega_{m}\right)$ - значение внутреннего поля сферы, отвечающего величине $\tilde{p}_{\mathrm{ph}}\left(\omega_{m}\right)$. Как следует из $(11)$, при снижении $E_{0}$, означающем уменьшение величины $p_{\mathrm{el}}$, будет происходить практически пропорциональное уменьшение $\xi$, поскольку снижение $\tilde{p}_{\text {рh }}$ будет осуществляться только через механизм роста $\omega_{m}$. При этом величина $\omega_{m} \tau_{\text {rel }}\left(\omega_{m} \tau_{r}\right)$ по мере снижения $E_{0}$ может существенно превысить единицу. Поэтому при вполне реальных значениях $\tau_{\text {rel }}$, отвечающих, например $\sigma_{s} \sim 10 \mathrm{~S} \cdot \mathrm{m}^{-1}$ и $\varepsilon_{s} \sim 10$, угловая скорость вращения может оказаться существенно выше $10^{11} \mathrm{~s}^{-1}$. Поскольку для сферы с $R \geq 0.1 \mu \mathrm{m}$, указанному значению $\omega_{m}$ отвечает линейная скорость, значительно превышающая $10^{4} \mathrm{~m} \cdot \mathrm{s}^{-1}$, возникает вопрос о реализуемости подобной скорости вращения частицы без ее механического разрушения.

\section{Динамика вращения несферической частицы}

Выше, как и в работе [1], предполагались выполненными три следующих экспериментальных условия:

1) форма частицы является идеально сферической;

2) материальный тензор второго ранга, описывающий такие физические параметры частицы, как коэффициент поглощения света и показатель преломления, проводимость, диэлектрическая проницаемость, отвечает симметрии скаляра;

3 ) показатели преломления частицы $n_{\text {par }}$ и диэлектрической жидкости $n_{\text {liq }}$ практически равны друг другу.

Как свидетельствуют результаты исследований вращения Квинке [7-10], нарушение первого условия может существенно повлиять на динамику движения частиц, в частности, устойчивость вращения. Ниже представлен краткий анализ динамики вращения частицы несферической формы для случая фотодиффузионного механизма генерации $\mathbf{p}_{\mathrm{ph}}$, но вначале остановимся на последствиях нарушения двух других условий.

1) Случай $n_{\text {par }} \neq n_{\text {liq. }}$ В силу симметрии сферической частицы, разница показателей преломления частицы и ее окружения может привести лишь к изменению величины $\mathbf{p}_{\text {ph }}$, но не его направления. Поэтому направление оси вращения частицы останется неизменной. Это же относится и к телам вращения, например, интересному, с точки зрения конструирования микро(нано)моторов, случаю частиц в форме цилиндра, когда его ось перпендикулярна $\mathbf{E}_{0}$.

2) Анизотропная сферическая частица. Следует отметить, что в данном случае неизбежно нарушение и третьего условия. Направление поля диффузии определяется направлением фотодиффузионного тока, коллинеарного $\mathbf{k}$ при любой ориентации частицы. Поэтому поворот сферы может привести лишь к изменению величины $p_{\text {ph }}$, и соответственно ее периодической модуляции при вращении частицы. Все сказанное справедливо и по отношению к частице цилиндрической формы, в случае указанной выше ориентации ее оси.

3) Изотропная частица несферической формы. Прежде всего, следует отметить, что при условии $n_{\text {par }}=n_{\text {liq }}$, вектор $\mathbf{p}_{\text {ph }} \| \mathbf{k}$ при любой ориентации частицы. Пусть частица имеет форму вытянутого эллипсоида вращения, у которого отношение $a=R_{l} / R_{s}$ близко к единице (здесь $R_{l}$ и $R_{s}$ - длина большой и малой полуосей эллипсоида соответственно). Рассмотрим вначале случай, когда большая ось эллипсоида лежит в плоскости образуемой векторами $\mathbf{k}$ и $\mathbf{E}_{0}$ (плоскость $\left(\mathbf{k}, \mathbf{E}_{0}\right)$ ). Тогда $\mathbf{T}_{\mathrm{ph}}$ направлен вдоль одной из малых осей, вращение вокруг которой можно описать с помощью уравнения, аналогичного (8):

$$
d \omega(t) / d t=\left(p_{p h}(t) / p_{s}\right) \omega_{m} \tau_{i}^{-1}-\omega(t) \tau_{i}^{-1},
$$

где $\omega_{m}-$ скорость стационарного вращения сферы с радиусом $\bar{R}=1 / 2\left(R_{l}+R_{s}\right)$, значения $p_{s}, \gamma$, также отвечают $\bar{R}$, а $\tau_{i}=J_{s} / \gamma$, где $J_{s}-$ момент инерции относительно малой оси эллипсоида. Учитывая небольшое отклонение величины $a$ от единицы, в условиях стационарного вращения зависимость $p_{\mathrm{ph}}(t)$ можно представить двумя первыми членами ее разложения в ряд Фурье

$$
p_{\mathrm{ph}}(t)=p_{s}+\delta p \sin \left(2 \omega_{m} t+\zeta\right),
$$

где $\xi-$ фаза, зависящая от начальной ориентации большой оси эллипсоида и $\delta p \ll p_{\text {sph. }}$. Аналогичным образом можно представить и зависимость $\omega(t)$

$$
\omega(t)=\omega_{m}+\delta \omega \sin \left(2 \omega_{m} t+\xi+\chi\right),
$$

где $\delta \omega \ll \omega_{m}$, а дополнительный фазовый сдвиг $\chi$ обусловлен инерцией вращения. Теперь, подставляя (13) 
и (14) в уравнение (12), получаем следующее равенство, определяющее условия стационарного вращения эллипсоида:

$$
\begin{aligned}
& 2 \delta \omega \omega_{m} \cos 2 \omega_{m} t-\left(\delta \omega / \tau_{i}\right) \sin 2 \omega_{m} t \\
& +\left(\delta p \omega_{m} / p_{s} \tau_{i}\right) \sin \left(2 \omega_{m} t-\chi\right)=0 .
\end{aligned}
$$

Равенство (15) требует выполнения следующих соотношений (при $\left.\omega_{m} \tau \ll 1\right)$ :

$$
\begin{gathered}
\delta \omega \approx \omega_{m}\left(\delta p / p_{s}\right), \\
\chi \approx 2 \omega_{m} \tau_{i} .
\end{gathered}
$$

Таким образом, реализуется устойчивое вращение эллипсоида с относительно небольшой периодической модуляцией угловой скорости. При произвольной начальной ориентации эллипсоида его вращение будет происходить аналогичным образом. Единственное отличие - это изменение амплитуды модуляции $p_{\mathrm{ph}}$ и $\omega_{m}$, т.е. величины $\delta p$ и $\delta \omega$, которые в предельном случае вращения вокруг большой оси эллипсоида будут равны нулю.

Здесь следует отметить, что вращающийся эллипсоид, в общем случае, представляет собой практически классический диполь Герца, способный генерировать электромагнитную волну (на частоте $2 \omega_{m}$ и кратных частотах) в результате периодического изменения величины $p_{\mathrm{ph}}$.

Продемонстрированная выше устойчивость вращения эллипсоида является следствием того, что оно должно рассматриваться, как случай вращения вокруг закрепленной оси. Однако при одновременном нарушении условий п. 2 и/или п. 3 ситуация меняется. Теперь y $\mathbf{p}_{\mathrm{ph}}$ могут появиться, в общем случае, компоненты, ортогональные вектору $\mathbf{k}$, одна из которых будет ортогональна, а вторая коллинеарна $\mathbf{E}_{0}$. С первой из них связано появление дополнительного вращательного момента ортогонального $\mathbf{T}_{\mathrm{ph}}$, который может проявить себя при вращении частицы в форме нутация оси вращения в плоскости, перпендикулярной $\left(\mathbf{k}, \mathbf{E}_{0}\right)$. В принципе, это может стать причиной неустойчивости вращения частицы при ее произвольной начальной ориентации, как это наблюдается в случае вращения Квинке [8-10]. Однако детальный анализ динамики вращения частицы при одновременном нарушении всех вышеуказанных условий выходит за рамки настоящей работы. Отметим также, что, как следует из процитированных работ [8-10], теоретические осложнения неизбежно возникнут при описании неадиабатического вращения частиц несферической формы, даже в случае выполнения условий п. 2 и п. 3.

\section{Заключение}

В настоящей работе рассмотрена динамика эффекта фотоиндуцированного вращения в постоянном однородном электрическом поле сферической частицы. В общем случае детальное теоретическое описание враще- ния требует решения задачи об установлении дрейфодиффузионного равновесия внутри освещаемой сферы в условиях ее вращения. Режим адиабатического вращения реализуется только при наличии механического сопротивления со стороны окружающей среды, в отсутствии которого неизбежен переход к неадиабатическому вращению. При этом решающее влияние на динамику неадиабатического вращения оказывает соотношение между величиной электро- и фотоиндуцированных дипольных моментов. Полученные результаты позволяют предположить, что фотоиндуцированное вращение частиц несферической формы (безотносительно к режиму вращения) может послужить основой для создания генератора электромагнитных волн, а также использоваться для экспериментального наблюдения собственно самого эффекта.

\section{Список литературы}

[1] Грачев А.И. // ФТТ. 2018. Т. 60. Вып. 4. С. 666-669.

[2] Quincke G. // Ann. Phys. Chem. Band. 1896. Vol. 59. P. 417.

[3] Jones T.B. // IEEE Trans. Ind. Appl. 1984. Vol. IA-20. P. 845.

[4] Turku I. // J. Phys. A: Math. Gen.1987. Vol. 20. P. 3301.

[5] Gharghi M., Bai H., Stevens G., Sivoththaman S. // IEEE Trans. Elec. Dev. 2006. Vol. 53. P. 1355.

[6] Davis L.C. // J. Appl. Phys. 1993. Vol. 73. P. 680.

[7] Jones T.B. Electromechanics of Particles. Cambridge, N.Y: Cambridge University Press, 1995.

[8] Cẽbers A., Lemaire E., Lobry L. // Phys. Rev. E. 2001. Vol. 63. P. 016301.

[9] Dolinsky Yu., Elperin T. // Phys. Rev. E. 2005. Vol. 71. P. 056611.

[10] Brosseau Q., Hickey G., Vlahovska P.M. // Phys. Rev. Fluid. 2017. Vol. 2. P. 014101. 